Results There were 600 cases of lung cancer in the highest exposed group and 668 in the lowest exposed group. The relative risk comparing high and low exposed decreased the years after exposure had stopped. It was 1.8 during the decade when the exposure come to an end, decreased to 1.5 (95\% CI 1.31.9) the next decade, to 1.2 (95\% CI 1.0-1.5) the decade later and finally to $1.0(95 \% \mathrm{CI} 0.8-1.2)$ three decades after the exposure to asbestos had stopped.

Conclusions The time pattern of asbestos related lung cancer seem to follow a similar pattern as the risk of lung cancer in exsmokers.

\section{ASSOCIATION OF OCCUPATIONAL TRAJECTORIES WITH ALCOHOL USE DISORDERS IN A LONGITUDINAL NATIONAL SURVEY}

${ }^{1}$ John D Meyer, ${ }^{2}$ Miriam Mutambudzi. ' ${ }^{1}$ cahn-Mount Sinai School of Medicine, New York,

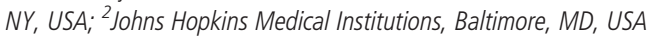

\subsection{6/oemed-2014-102362.14}

Objectives We posited mutually-reinforcing longitudinal pathways between occupation and alcohol use disorders (AUD).

Method Longitudinal trajectories of work substantive complexity were constructed by growth mixture modelling (GMM) of occupational data from the National Longitudinal Survey of Youth 1979 and O*NET work variables. AUD was determined using a set of 25 questions that map onto 9 criteria for alcohol dependence. Prevalent AUD was one occurred at or before the first alcohol questionnanire in 1989; an incident AUD was a new case between 1989 and the 1994 survey. The association between work trajectories and AUD was modelled using Poisson regression in a generalised linear model adjusting for covariates. Results Lower work trajectories were associated with higher AUD prevalence (prevalence ratio 1.41; 95\% confidence interval 1.041.91 for lowest versus highest class). Incident AUDs were associated with risk of a decline in work trajectory class in both high $(\mathrm{OR}=2.68 ; 95 \%$ CI $1.34-5.35)$ and low $(\mathrm{OR}=1.62$ 95\% CI 1.01-2.60) initial classes. Interval educational attainment was not associated with AUD within a specific occupaitonal trajectory class Conclusions Low occupational trajectories are associated with increased AUD prevalence. Incident AUDs may be followed by a decline in work SC. These findings suggest self-reinforcing relations between the development of AUD and both prior and subsequent work.

\section{PATTERNS OF SITE-EMPLOYMENT OF CONSTRUCTION WORKERS ON AND OFF COMMERCIAL CONSTRUCTION SITES IN NEW ENGLAND AND THE RELATIONSHIP TO MUSCULOSKELETAL PAIN}

${ }^{1}$ Emily H Sparer, ${ }^{1}$ Cassandra A Okechukwu, ${ }^{2} J u s t i n$ Manjourides, ${ }^{1}$ Robert F Herrick, 1,3 Jeffrey N Katz, ${ }^{1,2}$ Jack T Dennerlein. ' Harvard School of Public Health, Boston, MA, USA; ${ }^{2}$ Northeastern University, Boston, MA, USA; ${ }^{3}$ Brigham and Women's Hospital, Boston, MA, USA

\subsection{6/oemed-2014-102362.15}

Objectives Construction workers who work on multiple jobsites have a high prevalence of musculoskeletal disorders. Yet, scant quantitative information exists in the scientific literature on the relationship between worksite mobility patterns and musculoskeletal disorders.

Method Self-reported musculoskeletal pain, as defined as pain experienced in one of seven body areas in the past month, work history, and demographics were collected from 776 Boston area workers on their first day at one of seven commercial construction projects. Workers were classified as long-term workers (onsite greater than or equal to 30 days) or short-term workers (less than 30 days). Bivariate and multiple logistic regression analyses tested the relationship between term length and prevalence of self-reported musculoskeletal pain, adjusting for relevant covariates.

Results Of the 776 new workers, 344 (44\%) were on-site after one month, 164 (21\%) remained after two months, and only 74 $(10 \%)$ remained after three months. Thirty-three percent of workers reported musculoskeletal pain at baseline. Short-term workers were 2.02 times (95\% CI: $1.32,3.08)$ more likely to report any musculoskeletal pain at baseline than long-term workers, when controlling for trade and tenure. Reporting of singleand multi-site pain was also associated with term length, with statistically significant adjusted odds ratios of 2.00 and 2.35, respectively.

Conclusions The observed excess of self-reported pain in shortterm workers when compared to long-term workers mirrors disparities between temporary and non-temporary workers in other industries. This observed effect highlights the need to consider worksite mobility when analysing and interpreting data aimed at improving construction worker health and safety.

\section{COLORECTAL CANCER RISK AND SHIFT WORK IN A POPULATION-BASED CASE-CONTROL STUDY IN SPAIN (MCC-SPAIN)}

${ }^{1,2}$ Kyriaki Papantoniou, ${ }^{1,2}$ Manolis Kogevinas, ${ }^{2,3}$ Vicente Martin Sanchez, ${ }^{2,4}$ Victor Moreno, ${ }^{2,5}$ Marina Pollan, ${ }^{2,6}$ Jose Juan Jimenez Moleón, ${ }^{2,7}$ Eva Ardanaz, ${ }^{2,8}$ Jone MAltzibar, ${ }^{2,9}$ Rosana Peiro, ${ }^{2,10}$ Adonina Tardon, ${ }^{2,11}$ Juan Alguazil, ${ }^{2,12}$ Carmen Navarro, ${ }^{2,13}$ Ines Gomez-Acebo, ${ }^{1,2}$ Gemma Castano-Vimyals. ${ }^{1}$ Centre for Research in Environmental Epidemiology (CREAL, Barcelona, Spain; ${ }^{2}$ CIBER Epidemiologia Y Salud Publica (CIBERESP), Madrid, Spain; ${ }^{3}$ Universidad de León, Leon, Spain; ${ }^{4}$ Catalan Institute of Oncology, Hospitalet, Spain; ${ }^{5}$ Centro Nacional de Epidemiología-Instituto de Salud Carlos III, Madrid, Spain; ${ }^{6}$ Universidad de Granada, Granada, Spain; ${ }^{7}$ Servicio Navarro de Salud-Instituto de Salud Publica de Navarra, Pamplona, Spain; ${ }^{8}$ Subdirección de Salud Pública de Gipuzkoa, Donostia, Spain; ${ }^{9}$ Centre for Research in Public Health, Valencia, Spain; ${ }^{10}$ Instituto Universitario de Oncología, Universiidad de Oviedo, Oviedo, Spain; ${ }^{11}$ Universiidad de Huelva, Huelva, Spain; ${ }^{12}$ Servicio Epidemiología, Consejería Sanidad, Murcia, Spain; ${ }^{13}$ Universidad de Cantabria, Santander, Spain

\subsection{6/oemed-2014-102362.16}

Objectives Epidemiological cancer studies on shift work have focused on breast cancer while evidence on other tumours is limited. We evaluated colorectal cancer risk in relation to night and rotating shift work and genetic variation, in a population based case-control study in Spain.

Method 1066 male and 592 female incident colorectal cancer cases and 3388 randomly selected population controls of both sexes, enrolled in 11 regions of Spain, were included. Information was collected on socio-demographic, lifestyle, medical history and other variables by face-to-face interviews. Lifetime occupational history on daily time schedule of each job, day/night/rotating shifts, light at night exposure, and duration of different jobs, was used for exposure assessment. We used unconditional logistic regression adjusting for potential confounders.

Results Among controls 10\% of males and 4\% females had ever worked full time in permanent night shifts (working between midnight and $6 \mathrm{am}$ ) and $24 \%$ of males and $14 \%$ of females in rotating shifts for $\geq 1$ year. Having ever performed rotating shift work was associated with an increased risk for colorectal cancer 